\title{
Tratamento cirúrgico de fratura mandibular decorrente de projétil de arma de fogo: relato de caso
}

\author{
Surgical treatment of mandibular fracture after gunshot injury: case report \\ Tratamiento quirúrgico de la fractura mandibular secundaria \\ a herida por arma de fuego: reporte de caso \\ Patrícia Rota BERMEJO \\ Juliana Zorzi COLÉTE \\ Gustavo Antonio Correa MOMESSO \\ Paulo Afonso de OLIVEIRA \\ José Hicsy FONSECA \\ Elio Hitoshi SHINOHARA
}

Programa de Pós-Graduação em Odontologia,Faculdade de Odontologia de Araçatuba, UNESP-Univ Estadual Paulista, Araçatuba-SP, Brasil

\begin{abstract}
Resumo
As lesões faciais causadas por arma de fogo ainda são motivo de muita preocupação no âmbito da saúde pública e podem causar grandes prejuízos estéticos e funcionais para o paciente, além de perda de qualidade de vida. Sendo assim, o objetivo deste estudo é relatar um caso clínico de paciente do sexo masculino, 39 anos, encaminhado por policiais militares à equipe de cirurgia e traumatologia bucomaxilofacial para avaliação de lesão facial apresentando trauma em face decorrente de projétil de arma de fogo, foi submetido à cirurgia para a remoção do projétil e reconstrução maxilofacial em ambiente hospitalar, sob anestesia geral, devido às condições sistêmicas do paciente. Foi removida a jaqueta do projétil alojada em mucosa jugal direita e fragmentos dentários que se encontravam na linha de trajeto do projétil. Notou-se mobilidade do segmento anterior da mandíbula e lacerações em mucosa oral interna. No quarto dia foi realizada glossectomia parcial, em região de terços anterior e médio e fixação de fratura. Optou-se pelo acesso extraoral, realizou-se remoção de fragmentos ósseos, redução da fratura em sínfise, instalação de placa de reconstrução do lado esquerdo e placa e parafusos de 2,0 mm em sínfise, sendo os parafusos bicorticais em zona compressiva e monocorticais em zona de tração. Foi possível concluir que a redução aberta com fixação com placas e parafusos é uma boa opção de tratamento para fraturas cominutivas causadas por projéteis de arma de fogo, devolvendo ao paciente aspectos estéticos, funcionais e psicológicos.

Descritores: Ferimentos por Arma de Fogo; Fraturas Mandibulares; Glossectomia.
\end{abstract}

\begin{abstract}
The gunshot injury is still being reason of concern to world public health. They can cause great aesthetic and functional damages to the patient, besides loss of quality of life. Thus, the aim of this study is report a case of a 39-year-old male patient, referred by military polices to the Oral and Maxillofacial Surgery team to evaluate a facial injury by firearm gunshot, showing facial trauma and comminuted mandibular fracture. It was undergoing to the surgical removal of the bullet and maxillofacial reconstruction performed under general anesthesia, due the systemic conditions of the patient. It was removed the projectile housed in the right buccal mucosa and tooth fragments found in projectile path line. It was observed mobility of the mandible anterior segment and lacerations on intern oral mucosa. At fourth day, it was performed a partial glossectomy of anterior and middle third and fracture fixation. It was choose the extra-oral approach and performed the removal of bone fragments, reduction of symphysis fracture, installation of a reconstruction plate on the left side and bicortical plates and screws of $2.0 \mathrm{~mm}$ system on the compressive area and monocortical on the traction area of the symphysis fracture. It is possible to conclude that the open reduction with plates and screws fixation it is a good choice of treatment to comminuted fractures caused by gunshot injury. This technique cans development aesthetic, function and self-esteem to patient

Descriptors: Wounds, Gunshot; Mandibular Fractures; Glossectomy.
\end{abstract}

\section{Resumen}

Las lesiones faciales por armas de fuego siguen siendo objeto de gran preocupación en la salud pública y pueden causar daños importantes estéticos y funcionales para el paciente, así como la pérdida de calidad de vida. Por lo tanto, el objetivo de este estudio es presentar un caso de un paciente de sexo masculino, de 39 años, enviado por la policía militar para el equipo quirúrgico y de trauma maxilofacial para la evaluación de daños faciales por proyectil de arma de fuego, y se sometió a una cirugía para extraer el proyectil y la reconstrucción maxilofacial realizado en un hospital bajo anestesia general debido a las condiciones sistémicas del paciente. El casquillo de una bala alojada en la mucosa bucal derecha y fragmentos dentales estaban en la línea de recorrido del proyectil fue eliminado. Se tomó nota de la movilidad del segmento anterior de la mandíbula y laceraciones en mucosa oral interna. En el cuarto día fue realizado glosectomía parcial en la región de los tercios anterior y medio y la fijación de la fractura. Elegimos el acceso extra oral realizado por la eliminación de fragmentos de hueso, la reducción e instalación de la placa de reconstrucción de la fractura de sínfisis en el lado izquierdo y placa y tornillos de 2,0 mm en la propia sínfisis, siendo los tornillos bicorticales en zona de compresión y mono cortical en la zona de tracción. Se concluyó que la reducción abierta con fijación con placas y tornillos es una buena opción de tratamiento para las fracturas conminutas causadas por proyectiles de armas de fuego.

Descriptores: Heridas por Arma de Fuego; Fracturas Mandibulares; Glosectomía.

\section{INTRODUÇÃO}

Apesar da incidência de crimes no mundo estarem diminuindo, as lesões causadas por arma de fogo continuam sendo um importante problema de saúde pública relacionado à significante taxa de morbidade e mortalidade, além de gastos excessivos para a sociedade. Os tratamentos dessas injúrias têm sido descritos na literatura da cirurgia plástica, 
cirurgia e traumatologia bucomaxilofacial e otorrinolaringologia ${ }^{1-4}$.

As lesões faciais advindas de arma de fogo podem resultar em injúrias severas que variam de acordo com o calibre da arma utilizada e da distância em que o paciente se encontra do local do disparo. Distâncias reduzidas, ferimentos provindos de projéteis com alta velocidade e ferimentos causados por espingardas podem resultar em consequências estéticas e funcionais devastadoras ${ }^{5}$.

$\mathrm{O}$ manejamento destes pacientes ainda permanece bastante controvérsio em relação à reconstrução cirúrgica. Alguns autores aconselham em realizar intervenção agressiva no estágio inicial da reconstrução de todas as estruturas envolvidas ${ }^{1,6-9}$. No entanto vários estudos relatam uma abordagem mais conservadora, sem a realização de procedimentos cirúrgicos ${ }^{10-15}$.

As técnicas mais discutidas atualmente no manejamento destas lesões são a fixação maxilomandibular, fixação com fio intra-ósseo ou fixação externa com pinos (May, 1973; Neupert, 1991; Walker, 1984). No entanto, a tecnologia da fixação interna rígida das placas de titânio é agora amplamente utilizada no tratamento tanto traumas faciais, tanto penetrantes quanto contundentes ${ }^{16}$.

\section{CASO CLÍNICO}

Paciente melanoderma, 39 anos, sexo masculino, foi trazido por policiais militares à equipe de Cirurgia e Traumatologia Bucomaxilofacial, após ter participado de uma troca de tiros em uma tentativa de fuga, após a realização de um assalto a mão armada. Os policiais relataram que a arma utilizada era de calibre ponto $40 \mathrm{~mm}$ e o tempo do ocorrido até o atendimento emergencial foi de aproximadamente trinta minutos.

Prontamente foi solicitada avaliação da equipe de Cirurgia e Traumatologia Bucomaxilofacial. O exame inicial diagnosticou presença de orifício de entrada do projetil em formato irregular, com áreas de chamuscamento de pele em região de ângulo e corpo da mandíbula esquerda, e orifício de saída, de diâmetro menor e aspecto elíptico na mesma direção do lado oposto (Figuras 1A e 1B). O paciente apresentava-se deambulante, consciente $\mathrm{e}$ orientado. Verificou-se a presença de ferimento lacerante em língua com reversão anterior de sua porção posterior com comprometimento da artéria lingual bilateral (Figura 1C), má oclusão, mobilidade de segmento anterior da mandíbula, crepitação e impossibilidade de fechamento da boca. A família colaborou com a anamnese e relatou ser o paciente portador do vírus HIV, fazendo uso do coquetel AZT para o tratamento.

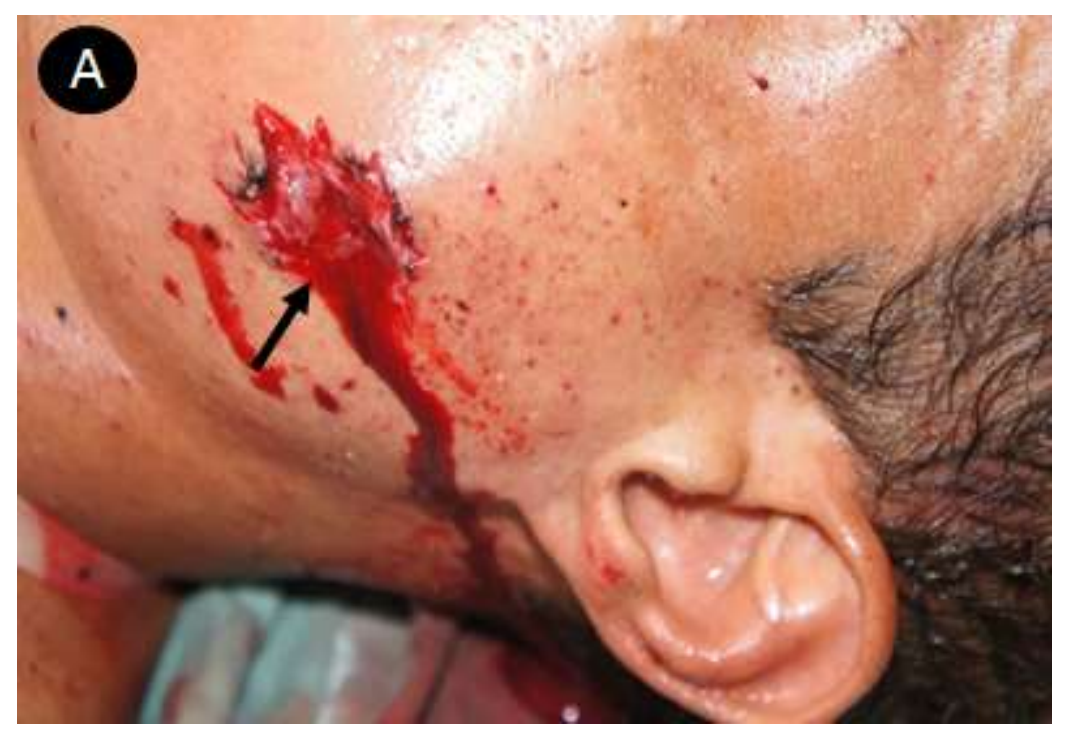

Figura 1A: Orifício de entrada do projétil.

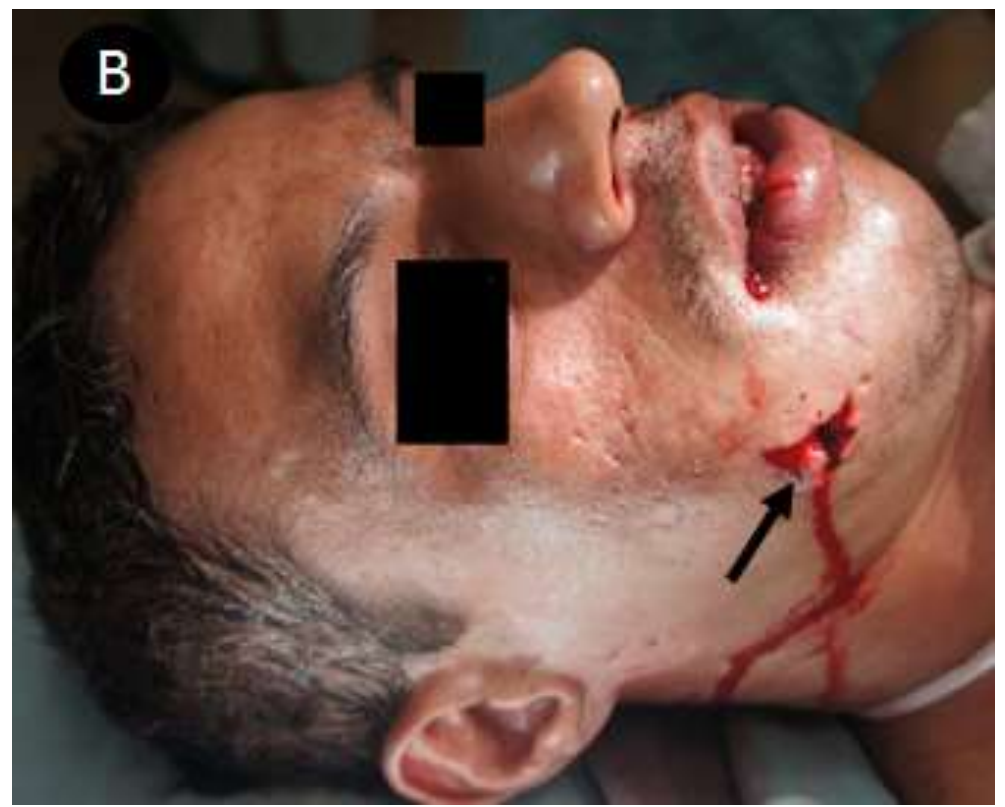

Figura 1B: Orifício de saída do projétil.

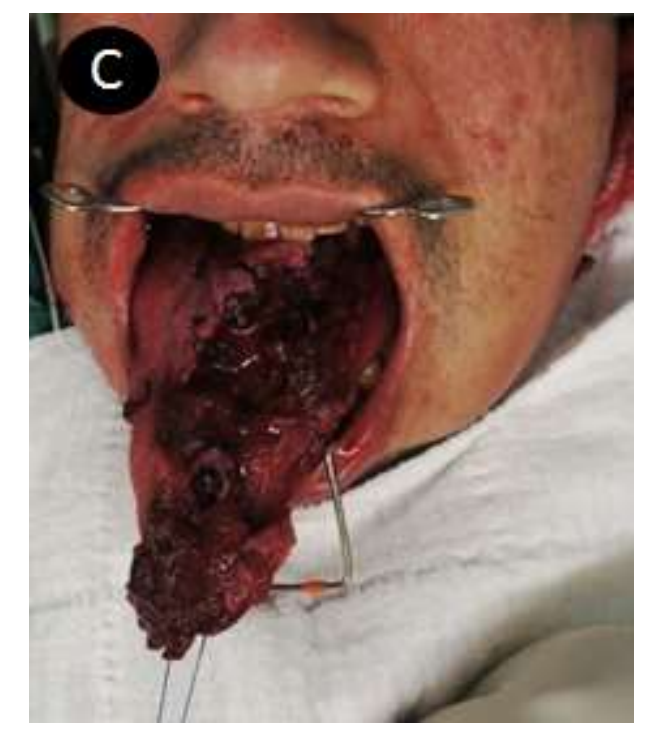

Figura 1C: Ferimento lacerante em língua com reversão anterior de sua porção posterior.

Devido ao edema em língua poder causar comprometimento de vias aéreas superiores, e ser um paciente além de imunocomprometido, oferecer risco para a equipe, optou-se pela realização de intervenção em centro cirúrgico e também de intubação por traqueostomia sob anestesia geral. Com o paciente devidamente intubado e anestesiado, foi feito o preparo do campo operatório e antissepsia intra e extraoral com digluconato de clorexidina. Localizou-se jaqueta do projétil alojada em mucosa jugal direita e sua remoção foi feita com pinça hemostática (Figuras 2A, 2B e 2C), sem dificuldades ou intercorrências. Também foram removidos fragmentos dentários que se encontravam na linha de trajeto do projétil e por isso sofreram fraturas. Também se notou mobilidade do segmento anterior da mandíbula e lacerações em mucosa oral interna. As bordas necróticas dos tecidos foram devidamente debridadas, os tecidos copiosamente irrigados com solução salina, feita sutura em massa em região posterior de língua para hemostasia da artéria lingual e reconstrução lingual com sutura, repetindo-se o mesmo procedimento para a mucosa bucal.

O paciente foi internado, foram pedidos os seguintes exames de imagem: Rx de Towne, Waters, Lateral oblíqua de mandíbula direita e esquerda, Póstero-anterior de mandíbula; assim como avaliação da clínica médica que solicitou exames laboratoriais para avaliação do estado de comprometimento da saúde geral do paciente. Foi prescrita terapia antibiótica e analgésica, além de dieta líquida e manutenção da traqueostomia.

Os exames laboratoriais e a avaliação da clínica médica revelaram condição geral de saúde estável e 
os exames de imagem revelaram cominuição de corpo e ângulo da mandíbula esquerda e fratura de síntese, situações já esperadas pelo exame clínico.

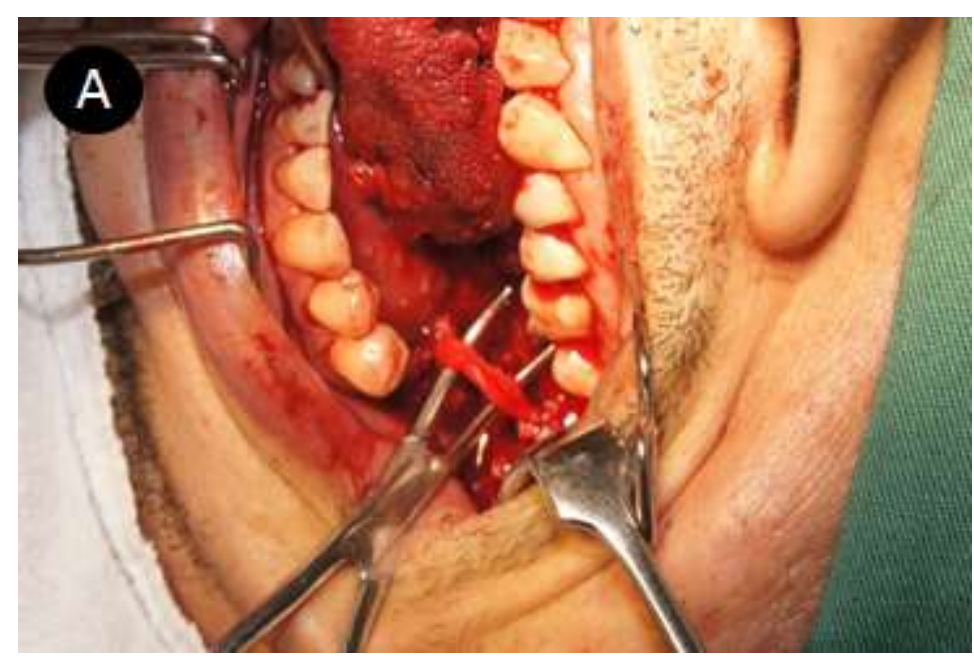

Figura 2A: Isolamento do nervo alveolar inferior durante remoção de projétil.

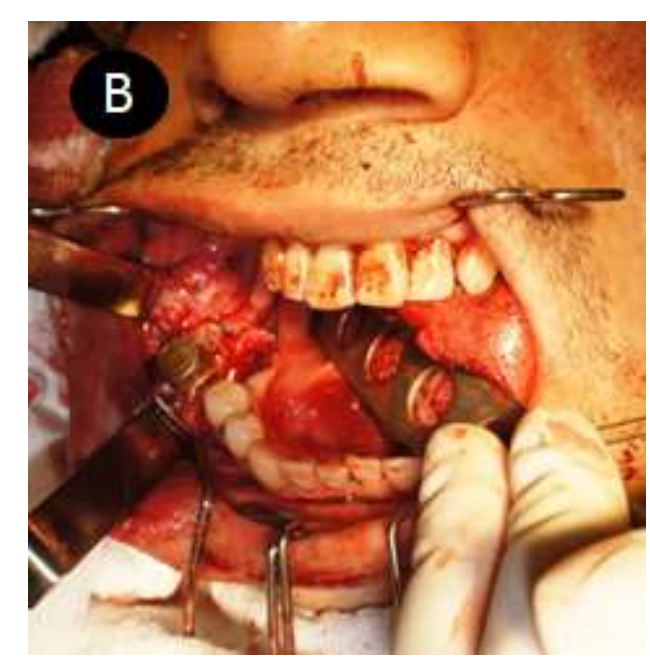

Figura 2B: Remoção de projétil em região superficial de mucosa jugal.

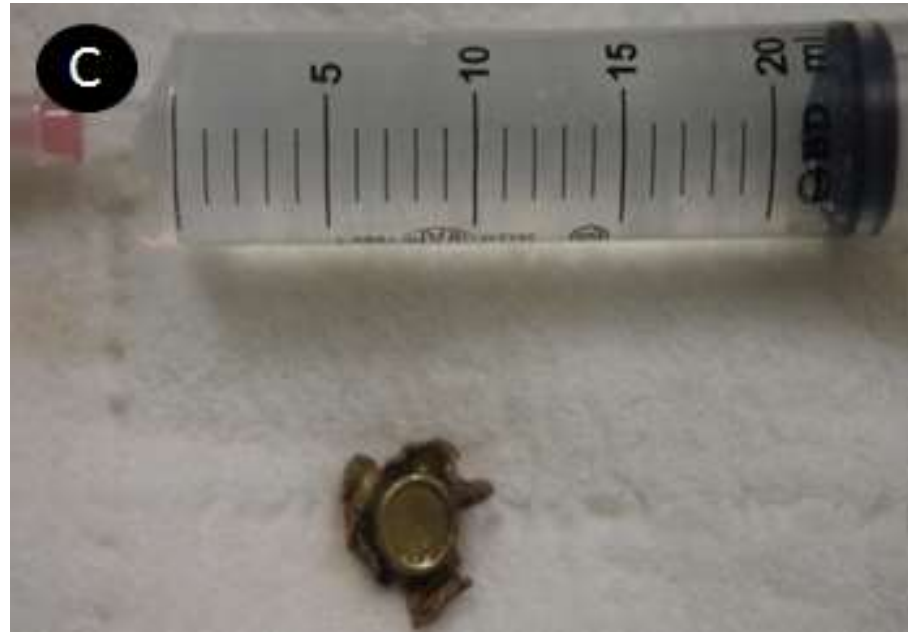

Figura 2C: Remoção de projétil em região superficial de mucosa jugal.

No quarto dia da internação o paciente apresentou áreas de necrose extensas em língua $\mathrm{e}$ aumento da sintomatologia dolorosa (Figuras 3A e 3B).

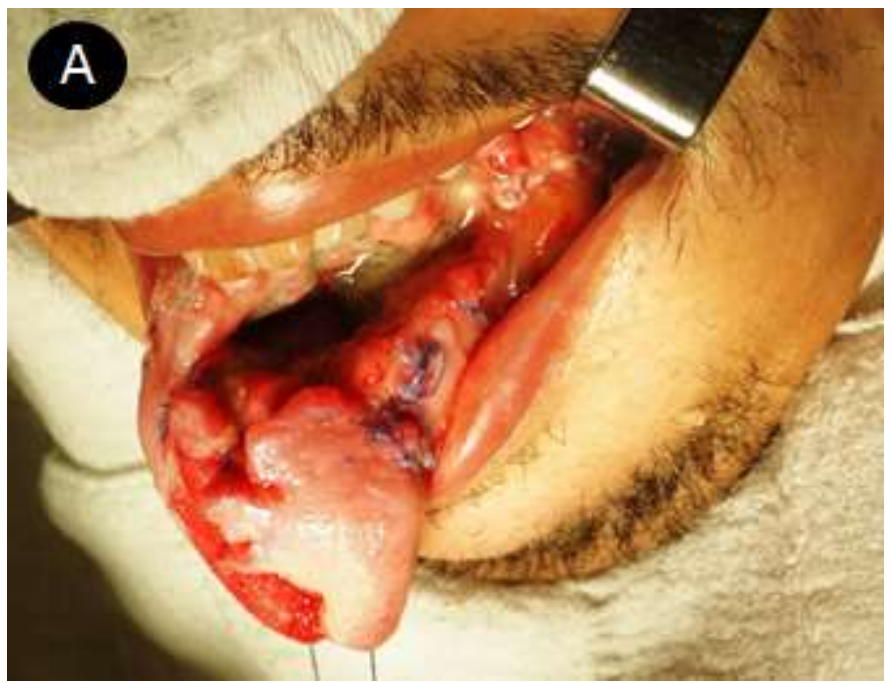

Figura 3A: Presença de infecção e necrose tecidual lingual no quarto dia de internação, devido à imunossupressão causada pela AIDS.

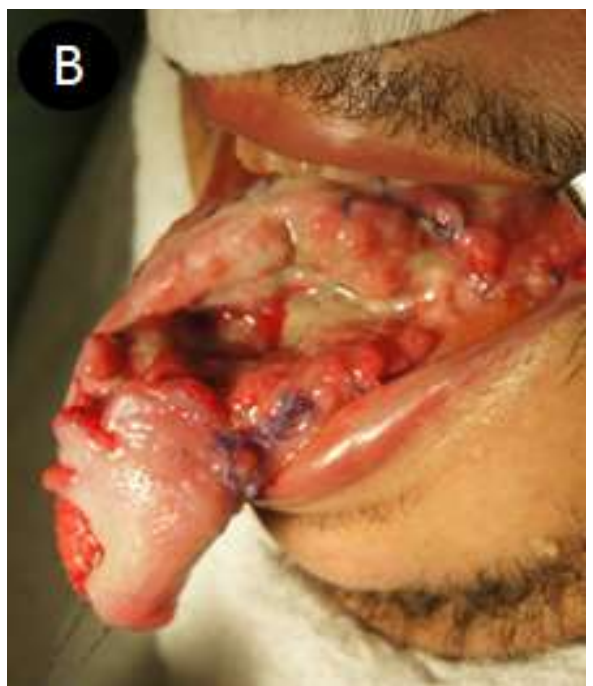

Figura 3B: Presença de infecção e necrose tecidual lingual no quarto dia de internação, devido à imunossupressão causada pela AIDS.

Nova intervenção em centro cirúrgico foi feita, dessa vez para realização de glossectomia parcial, em região de terços anterior e médio (Figuras 4A, 4B E 4C). A prescrição medicamentosa foi mantida, optando-se pelo uso concomitante de anti-inflamatório esteroidal e o paciente acompanhado para avaliar-se a regressão do quadro e determinação da oportunidade cirúrgica, a qual se estabeleceu após quatro dias.

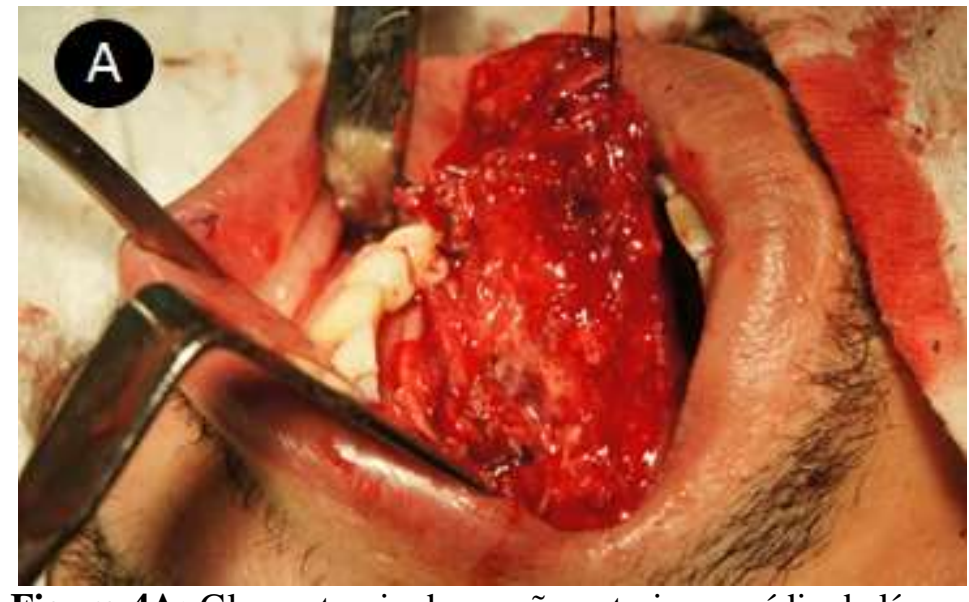

Figura 4A: Glossectomia da porção anterior e média da língua após presença de necrose tecidual.

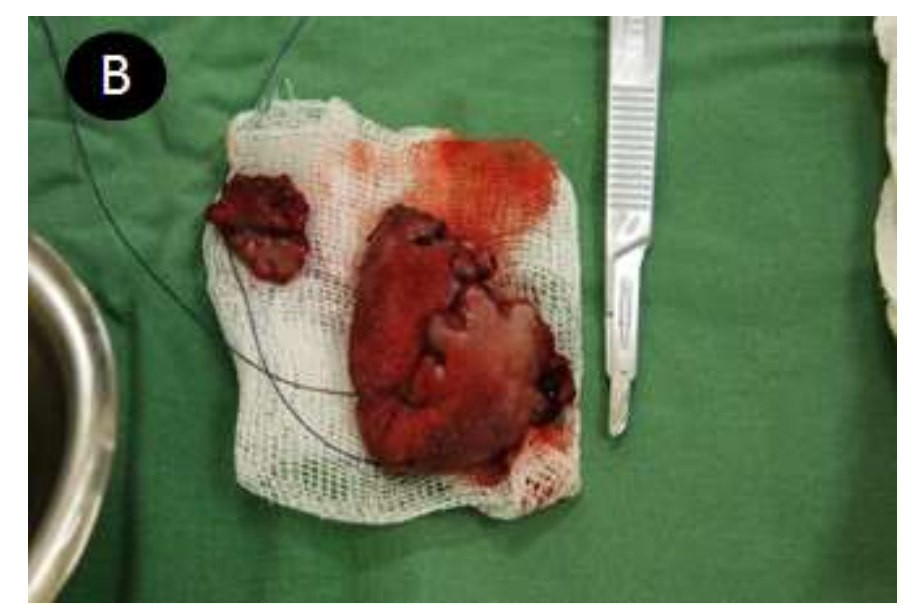

Figura 4B: Glossectomia da porção anterior e média da língua após presença de necrose tecidual.

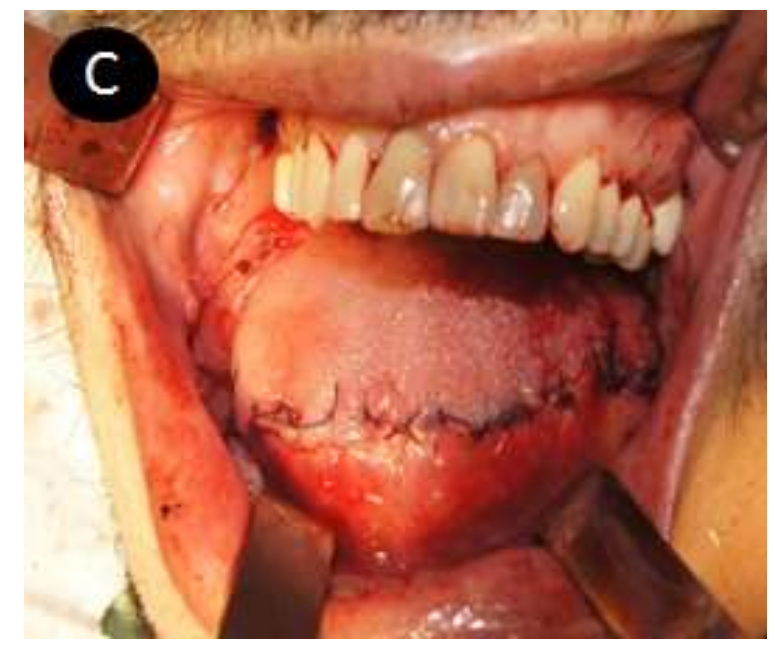

Figura 4C: Glossectomia da porção anterior e média da língua após presença de necrose tecidual. 
O paciente foi levado novamente ao centro cirúrgico para redução e fixação de fratura sob anestesia geral. Optouse pelo acesso extraoral para exposição das fraturas. Realizou-se remoção de fragmentos ósseos, redução da fratura em sínfise com pinça para osso, instalação de placa de reconstrução do lado esquerdo e placa e parafuso de 2,0 $\mathrm{mm}$ em sínfise, sendo os parafusos bicorticais em zona compressiva e monocorticais em zona de tração. A sutura foi feita por planos e o paciente recebeu bloqueio maxilomandibular (Figuras 5A, 5B, 5C, 5D, 5E e 5F).

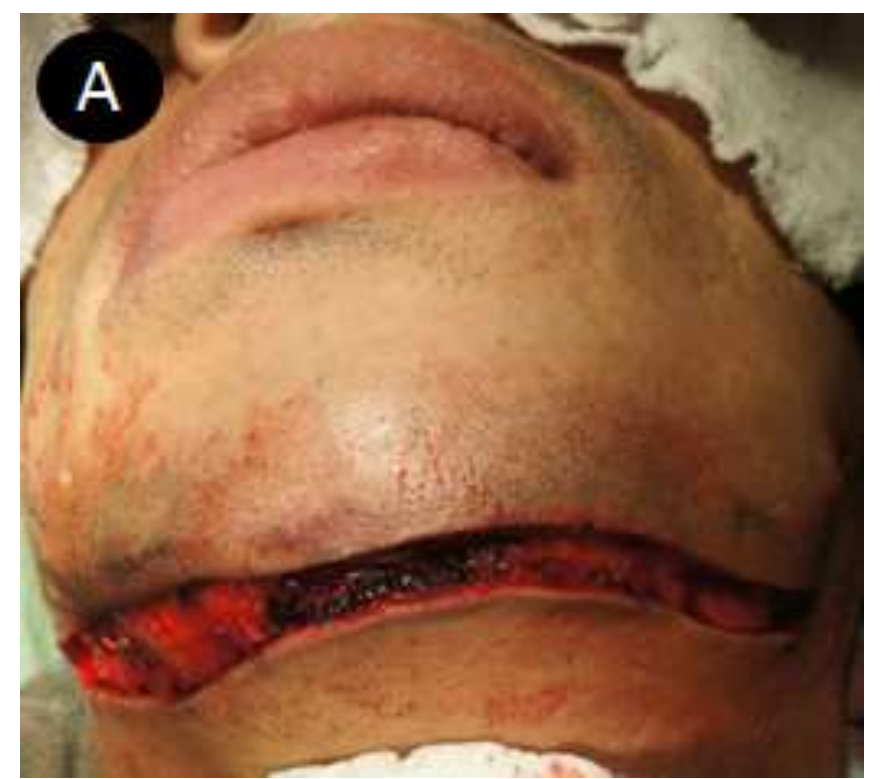

Figura 5A: Acesso submandibular para acesso das fraturas

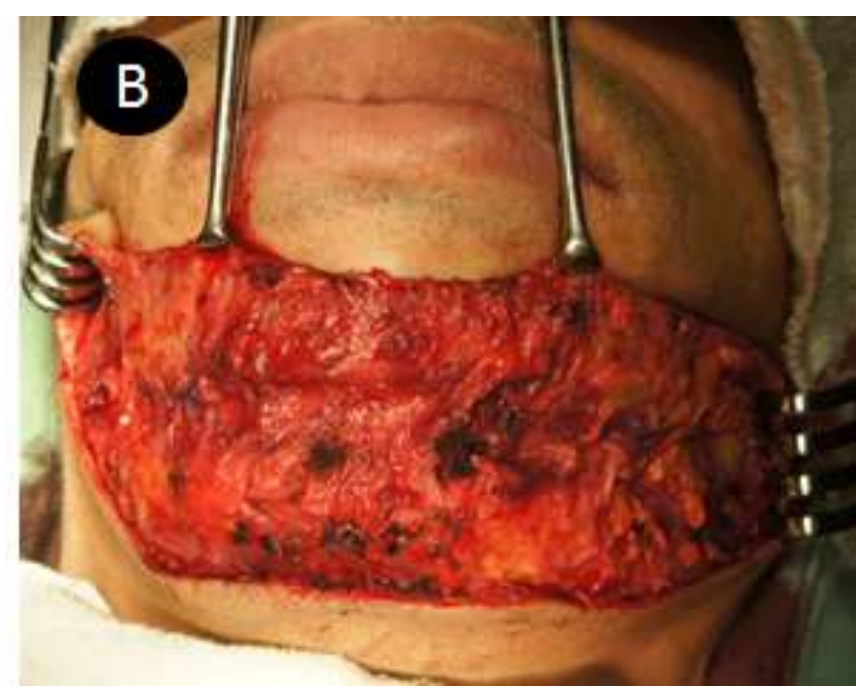

Figura 5B: Acesso submandibular para acesso das fraturas

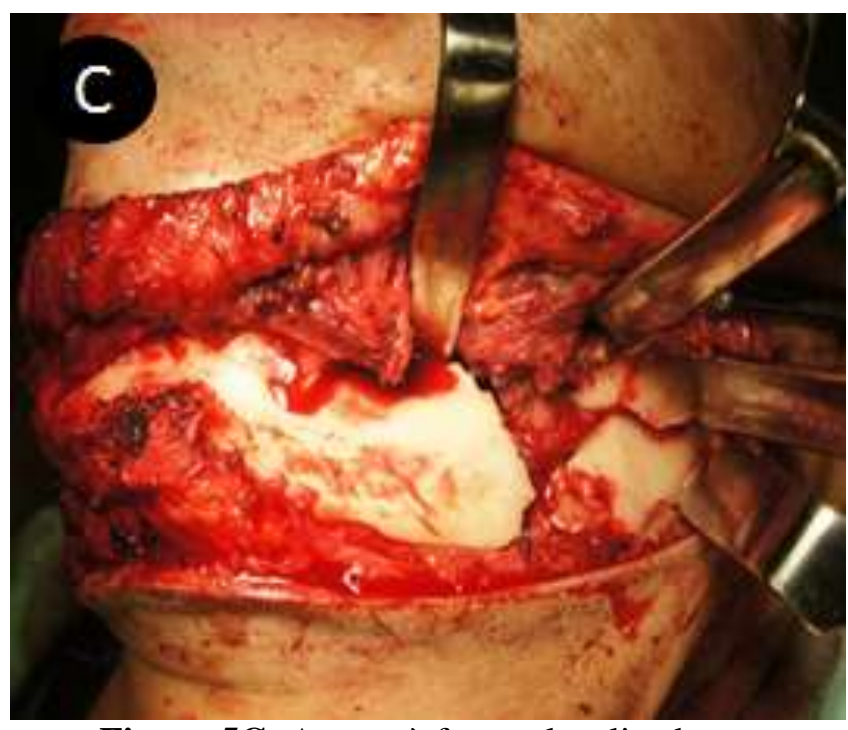

Figura 5C: Acesso à fratura localizada em ângulo mandibular esquerdo.

A antibioticoterapia foi mantida por mais três dias, quando o paciente recebeu alta. Como o paciente foi levado para encarceramento achou-se prudente manter o arco de Erich por mais 45 dias.

Após os 45 dias da alta hospitalar o paciente foi trazido ao ambulatório de Cirurgia e Traumatologia Bucomaxilofacial para acompanhamento e remoção do arco de Erich. Notou-se total reparação mucosa e óssea, sem sinais de mobilidade em sínfise mandibular, ou crepitação em qualquer outra região, assim como ausência de tecido necrótico.

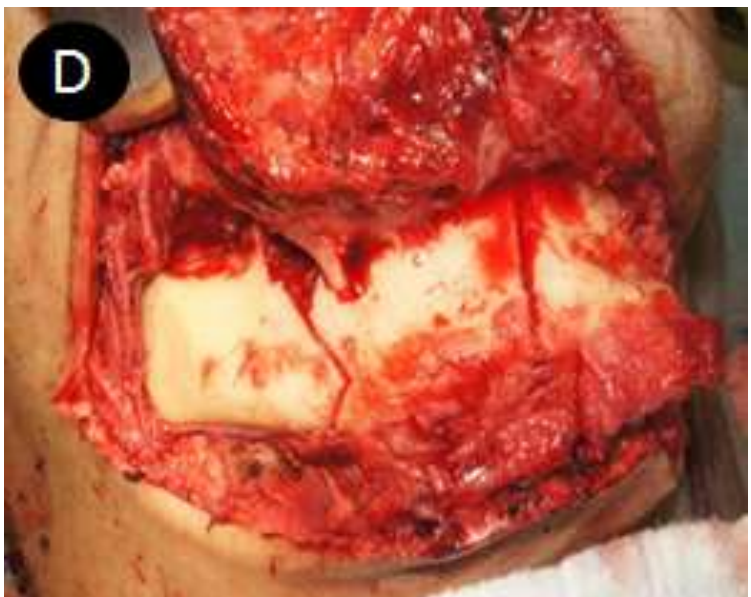

Figura 5D: Acesso à fratura localizada em região de sínfise mandibular.

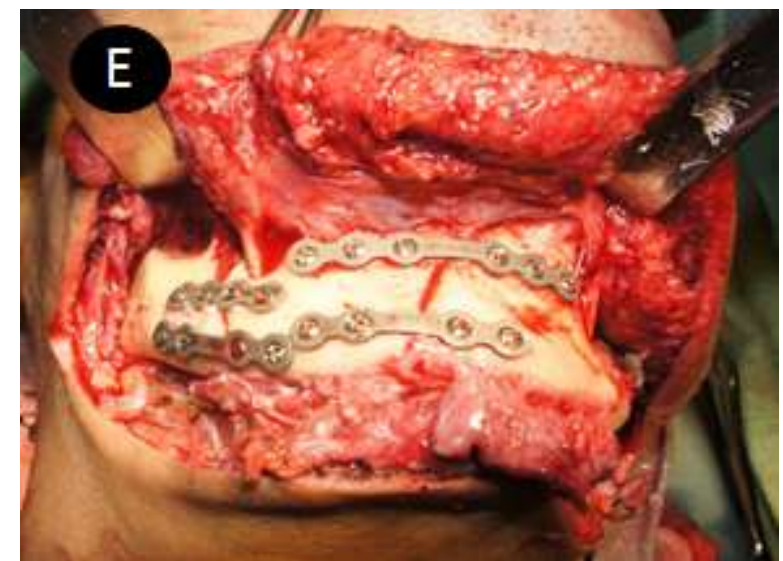

Figura 5E: Fixação de placas e parafusos do sistema $2.0 \mathrm{~mm}$ em zonas de tensão e compressão de sínfise mandibular.

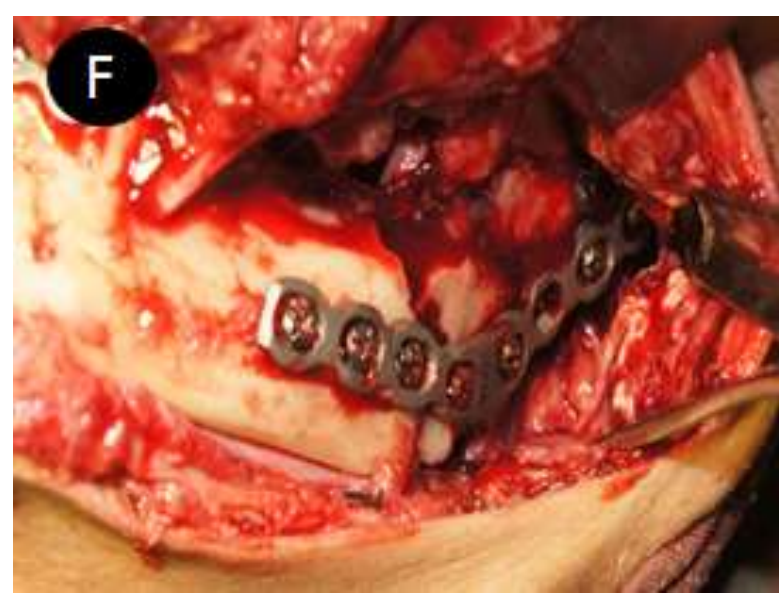

Figura 5F: Fixação de placa de reconstrução e parafusos do sistema $2.4 \mathrm{~mm}$ em região de ângulo mandibular esquerdo.

\section{DISCUSSÃO}

O uso de armas de fogo associado à criminalidade afeta a todos, independente da classe social. Estudos realizados em dois centros de Trauma, no sul dos EUA, constataram que $7 \%$ das admissões são vítimas de injúrias por arma de fogo e, dentre essas, $10 \%$ em face $^{17}$

As lesões maxilofaciais ocorriam principalmente por acidentes automobilísticos até meados da década de 90, mas hoje devido à facilidade da aquisição de armamentos e aumento da criminalidade, as lesões por armas de fogo na face, têm tido uma alta incidência nos grandes hospitais de trauma. As agressões por projéteis de arma de fogo em crianças, apesar de serem de baixa frequência, promovem lesões severas, principalmente quando atingem centros de crescimento $^{18,19}$.

Dos ossos da face, a mandíbula por ser um osso mais proeminente, apresenta um maior acometimento por lesões por arma de fogo, sendo o corpo mandibular o local mais acometido por este tipo de ferimento, conforme o caso descrito $^{20,21}$ 
Os ferimentos causados por armas de fogo caracterizam-se como feridas sujas, devido à possibilidade de contaminação não só pelo projétil em si como também pelos projéteis secundários originados durante o trajeto do mesmo durante o voo e ao atingir o alvo como, por exemplo, resquícios de chumbo e pólvora, fragmentos de tecido, do cartucho, dentes, espículas ósseas etc., e como tal devem receber cobertura antibiótica e antitetânica. Nesse caso foi utilizado Cefazolina1g, a cada 8 horas por via endovenosa.

$\mathrm{O}$ fato de o paciente haver apresentado necrose lingual pode ser atribuído não só a extensão e natureza do ferimento, mas também ao próprio quadro da AIDS, que deprime o sistema imune havendo falha nos processos de defesa natural humoral (pelas imunoglobulinas e o sistema complemento) do hospedeiro e possibilitando o crescimento da população de microrganismos oportunistas que permitem a instalação e progressão de processos sépticos.

Em estudo retrospectivo Hollier et al. ${ }^{5}$ constataram que a necessidade de intervenção cirúrgica apresentou alta frequência entre os casos analisados, sendo de 32\% de $76 \%$ dos casos. Além disso, os autores observaram que a maior parte dos casos que necessitava de procedimento cirúrgico era de fraturas de mandíbula, em que $76 \%$ dos pacientes foram submetidos à fixação interna rígida com a instalação de fixador externo ou fixação maxilomandibular.

Tem sido relatado que a fraturas ósseas advindas de disparos por arma de fogo devem ser tratadas de maneira conservadora, sem intervenções cirúrgicas ${ }^{22}$. No entanto, diversos estudos têm apontado para a conduta cirúrgica nesses pacientes ${ }^{23-25}$.

O tratamento escolhido para a fratura cominutiva apresentada foi a redução aberta de todos os fragmentos e a fixação com placas e parafusos do sistema $2.0 \mathrm{~mm}$ em sínfise e de reconstrução para a fratura de ângulo e corpo mandibular, sendo que a irrigação e inervação dos segmentos fraturados não foi comprometida, haja vista a integridade da mucosa oral e do nervo alveolar inferior.

A remoção do projétil deve ser considerada quando este estiver superficial, ou comprometendo a função da estrutura atingida, tiver proximidade com estruturas vitais ou quando o acesso a ele estiver dificultado. Nesses casos o projétil é sepultado e feito acompanhamento através de análises de imagem (tomografias computadorizadas, arteriografias digitais, etc.) se houver possibilidade de lesão a estruturas vitais, ou se puder lesionar um feixe vasculonervoso tardiamente por deslocamento do projétil pela movimentação muscular do local. Como o projétil no caso relatado estava superficialmente alojado em mucosa jugal, sem comprometimento de nenhuma estrutura nobre, foi facilmente removido.

Como o paciente foi trazido ao serviço pelos próprios policiais envolvidos na troca de tiros, foi possível obter informações quanto à natureza do disparo, sua distância e calibre da arma, possibilitando à equipe um conhecimento adicional no sentido de prever os danos teciduais causados no trauma e possíveis consequências, assim como plano de tratamento adequado.

\section{CONCLUSÃO}

A redução aberta com fixação interna rígida por meio de placas e parafusos é uma alternativa interessante para o tratamento de fraturas mandibulares provindas de disparo por arma de fogo e que esse tipo de lesão permanece como assunto importante no âmbito da saúde pública devido ao desafio no tratamento, além das sequelas funcionais, estéticas e psicológicas que permanecem ao longo da vida.

\section{REFERÊNCIAS}

1. Gruss JS, Antonyshyn O, Phillips JH. Early definitive bone and soft-tissue reconstruction of major gunshot wounds of the face. Plast Reconstr Surg. 1991 Mar; 87(3):436-50.

2. May M, Cutchavaree A, Chadaratana P, West J. Mandibular fractures from civilian gunshot wounds: a study of 20 cases. Laryngoscope. 1973 Jun; 83(6):96973.

3. Neupert EA, Boyd SB. Retrospective analysis of lowvelocity gunshot wounds to the mandible. Oral Surg Oral Med Oral Pathol. 1991 Oct; 72(4):383-7.

4. Walker RV, Frame JW. Civilian maxillo-facial gunshot injuries. Int J Oral Surg. 1984 Aug; 13(4):263-77.

5. Hollier L, Grantcharova EP, Kattash M. Facial gunshot wounds: a 4-year experience. J Oral Maxillofac Surg. 2001 Mar; 59(3):277-82.

6. Clark N, Birely B, Manson PN, Slezak S, Kolk CV, Robertson B, et al. High-energy ballistic and avulsive facial injuries: classification, patterns, and an algorithm for primary reconstruction. Plast Reconstr Surg. 1996 Sep; 98(4):583-601.

7. Chen AY, Stewart MG, Raup G. Penetrating injuries of the face. Otolaryngol Head Neck Surg. 1996 Nov; 115(5):464-70.

8. Thorne $\mathrm{CH}$. Gunshot wounds to the face. Current concepts. Clin Plast Surg. 1992 Jan; 19(1):233-44.

9. Mladick RA, Georgiade NG, Royer J. Immediate flap reconstruction for massive shotgun wound of face. Case report. Plast Reconstr Surg. 1970 Feb; 45(2):186-8.

10. Kihtir T, Ivatury RR, Simon RJ, Nassoura Z, Leban S. Early management of civilian gunshot wounds to the face. J Trauma. 1993 Oct;35(4):569-75;

11. Weider L, Hughes K, Ciarochi J, Dunn E. Early versus delayed repair of facial fractures in the multiply injured patient. Am Surg. 1999 Aug; 65(8):790-3.

12. Demetriades D, Chahwan S, Gomez H, Falabella A, Velmahos G, Yamashita D. Initial evaluation and management of gunshot wounds to the face. J Trauma. 1998 Jul; 45(1):39-41.

13. Williams CN, Cohen M, Schultz RC. Immediate and long-term management of gunshot wounds to the lower face. Plast Reconstr Surg. 1988 Sep; 82(3):433-9.

14. Siberchicot F, Pinsolle J, Majoufre C, Ballanger A, Gomez D, Caix P. Gunshot injuries of the face. Analysis of 165 cases and reevaluation of the primary treatment. Ann Chir Plast Esthet. 1998 Apr; 43(2):132-40.

15. MacFarlane C. Management of gunshot wounds: the Johannesburg experience. Int Surg. 1999 Apr-Jun; 84(2):93-8.

16. Denny AD, Sanger JR, Matloub HS, Yousif NJ. Selfinflicted midline facial gunshot wounds: the case for a combined craniofacial and microvascular team approach. Ann Plast Surg. 1992 Dec; 29(6):564-70.

17. Cunningham LL, Haug RH, Ford J. Firearm injuries to the maxillofacial region: an overview of current thoughts regarding demographics, pathophysiology, and management. J Oral Maxillofac Surg. 2003 Aug; 61(8):932-42.

18. Shaikh ZS, Worrall SF. Epidemiology of facial trauma in a sample of patients aged 1-18 years. Injury. 2002 Oct; 33(8):669-71.

19. Gassner R, Tuli T, Hächl O, Rudisch A, Ulmer H. Cranio-maxillofacial trauma: a 10 year review of 9,543 cases with 21,067 injuries. J Craniomaxillofac Surg. 2003 Feb; 31(1):51-61. 
20. Oikarinen K, Ignatius E, Kauppi H, Silvennoinen U. Mandibular fractures in northern Finland in the 1980s--a 10-year study. Br J Oral Maxillofac Surg. 1993 Feb; 31(1):23-7.

21. Adi M, Ogden GR, Chisholm DM. An analysis of mandibular fractures in Dundee, Scotland (1977 to 1985). Br J Oral Maxillofac Surg. 1990 Jun; 28(3):1949.

22. Dutra JAA, Dutra FKAA, Azevedo RA, Carneiro Jr B Avaliação do tratamento conservador de fratura de mandíbula. Rev Cir Traumatol Buco-Maxilo-fac. 2009; 9(2):89-96.

23. Giuliani G, Anile C, Massarelli M, Maira G. Management of complex craniofacial traumas. Rev Stomatol Chir Maxillofac. 1997 Nov;98 Suppl 1:100-2.

24. Greenberg AM. Management of facial fractures. N Y State Dent J. 1998 Mar; 64(3):42-7.

25. Gruss JS, Mackinnon SE, Kassel EE, Cooper PW. The role of primary bone grafting in complex craniomaxillofacial trauma. Plast Reconstr Surg. 1985 Jan; 75(1):17-24.

\section{CONFLITO DE INTERESSES}

Os autores declaram não haver conflitos de interesse.

\section{AUTOR PARA CORRESPONDÊNCIA}

Gustavo Antônio Correa Momesso

gustavomomesso@gmail.com

Submetido em 28/09/2016 Aceito em 13/10/2016 